\title{
The Beta-Transformation's Companion Map for Pisot or Salem Numbers and their Periodic Orbits
}

\author{
Bruno Melo Maia \\ bmaia@autonoma.pt \\ Universidade Autónoma de Lisboa \\ Rua Sta. Marta 47 \\ 1150-293 Lisboa, PORTUGAL
}

(Final version accepted 27 January 2017)

\begin{abstract}
The $\beta$-transformation of the unit interval is defined by $T_{\beta}(x):=\beta x(\bmod 1)$. Its eventually periodic points are a subset of $[0,1]$ intersected with the field extension $\mathbb{Q}(\beta)$.

If $\beta>1$ is an algebraic integer of degree $d>1$, then $\mathbb{Q}(\beta)$ is a $\mathbb{Q}$-vector space isomorphic to $\mathbb{Q}^{d}$, therefore the intersection of $[0,1]$ with $\mathbb{Q}(\beta)$ is isomorphic to a domain in $\mathbb{Q}^{d}$. The transformation from this domain which is conjugate to the $\beta$-transformation is called the companion map, given its connection to the companion matrix of $\beta$ 's minimal polynomial.

The companion map and the proposed notation provide a natural setting to reformulate a classic result concerning the set of periodic points of the $\beta$-transformation for Pisot numbers. It also allows to visualize orbits in a $d$-dimensional space.

Finally, we refer connections with arithmetic codings and symbolic representations of hyperbolic toral automorphisms.
\end{abstract}

Keywords: beta-transformation, companion matrix, Pisot, Salem, periodic orbit

2010 Mathematics Subject Classification: 37A45, 37C15, 37P35, 11K16

\section{Introduction}

In 1957, Rényi [1] generalized integer base numbering systems to non-integer basis, introducing the $\beta$-expansion, or base $\beta$ representation of real numbers. The symbols of the $\beta$-expansion of $x \in[0,1]$ are related to iteration of $x$ under the $\beta$-transformation $T_{\beta}(x):=\beta x(\bmod 1)$. The dynamics of the $\beta$-transformation is encoded as a one sided shift on a space of symbolic sequences (for further details, please refer to [2], [3] or [4]).

The points of $[0,1]$ having eventually periodic $\beta$-expansions are a subset of $\mathbb{Q}(\beta) \cap[0,1]$. Bertrand and Schmidt in [5] and [6] independently proved that if $\beta$ is a Pisot number (an algebraic integer with algebraic conjugates having modulus less than 1$)$, then $\operatorname{Per}\left(T_{\beta}\right)=$ $\mathbb{Q}(\beta) \cap[0,1]$.

The problem is much harder if $\beta$ is a Salem number (an algebraic integer with algebraic conjugates of modulus less than or equal to 1 , and at least one pair of them having modulus 1). The $\beta$-expansion of 1 is particularly important: whenever it is finite, we say that $\beta$ is a beta number (which is always the case for Pisot numbers). Boyd proved in [7] that every Salem number of degree 4 is a beta number. In [8], Boyd produced heuristics (based on random walks) supporting the fact that every Salem number of degree 6 should be a beta number, but the same should not happen for degrees greater than 6 . 
In [9] Thurston observes that for the Salem case of degree $d \geq 4$, the orbit of 1 resembles a random walk in $\mathbb{R}^{(d-2) / 2}$. It is well known that Brownian motion in dimension bigger than 2 is not recurrent. If this idea could be made rigorous, we would expect that Salem numbers of degree at least 8 are not beta numbers. This experimental observation also provides a potential explanation for Boyd's result for degree 4 Salem numbers, and his heuristic for degree 6 Salem numbers.

Section 2 sets the notation and recalls some definitions concerning $\beta$-transformations, Pisot and Salem numbers, minimal polynomials and companion matrices, which will be needed in the subsequent sections.

In Section 3 we introduce our main result. We observe that if $\beta$ is an algebraic integer of degree $d$, then $\mathbb{Q}(\beta)$ is $d$-dimensional $\mathbb{Q}$-vector space with a basis $1, \beta, \ldots, \beta^{d-1}$. This basis defines a coordinate isomorphism between $\mathbb{Q}^{d}$ and $\mathbb{Q}(\beta)$. We define a transformation on a subset of $\mathbb{Q}^{d}$ which is conjugate to the restriction of $T_{\beta}$ to $\mathbb{Q}(\beta) \cap[0,1]$. We call it the companion map because it is related to the companion matrix of the minimal polynomial of $\beta$. We describe the geometry of the domain of the companion map and establish connections with [10] and [11]. Some results in [3] were relevant for this construction.

Section 4 proposes a reformulation in our setting of the classic results from [6]. We also translate into our setting the special Pisot numbers of the type $\beta^{2}=n \beta+1$, referred by [6], and explain how the companion map naturally factors to the toral automorphism. As a consequence, the result in [6] concerning the period of orbits follows immediately.

\section{The $\beta$-transformation and the $\beta$-expansion}

For any real $x$, define its floor as $\lfloor x\rfloor:=\max \{m \in \mathbb{Z} \mid m \leq x\}$.

Definition 2.1: Let $\beta>1$ and $\beta \notin \mathbb{N}$. The $\beta$-transformation is

$$
\begin{aligned}
T_{\beta}:[0,1] & \longrightarrow[0,1] \\
x & \longmapsto \beta x-\lfloor\beta x\rfloor .
\end{aligned}
$$

Definition 2.2: The $\beta$-expansion of $x \in[0,1]$ is the sequence

$$
\left(a_{1}, a_{2}, a_{3}, \ldots\right), \text { with } a_{n}:=\left\lfloor\beta T_{\beta}^{n-1}(x)\right\rfloor .
$$

This sequence is a representation of $x$ in basis $\beta$, because $x=\sum_{n=1}^{\infty} \frac{a_{n}}{\beta^{n}}$.

Let $\operatorname{Per}\left(T_{\beta}\right)$ be the set of eventually periodic points for $T_{\beta}$, and $\mathbb{Q}(\beta)$ be the field extension of $\mathbb{Q}$ by adjoining $\beta$. It is known that $\operatorname{Per}\left(T_{\beta}\right) \subseteq \mathbb{Q}(\beta) \cap[0,1]$ ([see 6, p. 269]). In order to study $\operatorname{Per}\left(T_{\beta}\right)$, we consider the restriction of $T_{\beta}$ to $\mathbb{Q}(\beta) \cap[0,1]$. The eventually periodic points for $T_{\beta}$ are the numbers in $[0,1]$ having eventually periodic $\beta$-expansions.

Definition 2.3: $A n$ algebraic integer is a root of a minimal polynomial

$$
p(x)=x^{d}+c_{d-1} x^{d-1}+\ldots+c_{1} x+c_{0}, \quad c_{k} \in \mathbb{Z} .
$$


The companion matrix of $p(x)$ is

$$
\mathbf{C}=\left[\begin{array}{ccccc}
0 & 0 & \cdots & 0 & -c_{0} \\
1 & 0 & \ddots & \vdots & -c_{1} \\
0 & 1 & \ddots & 0 & \vdots \\
\vdots & \ddots & \ddots & 0 & \vdots \\
0 & \cdots & 0 & 1 & -c_{d-1}
\end{array}\right]
$$

Some authors define the companion matrix as the transpose of $\mathbf{C}$. The eigenvalues of $\mathbf{C}$ are the roots of $p(x)$, because its characteristic polynomial is $(-1)^{d} p(\lambda)$ (see [12, p. 339]).

Let us represent vectors in $\mathbb{R}^{d}$ as column matrices, and their transposes as row matrices. The row vector $\boldsymbol{\beta}^{T}:=\left[\begin{array}{llll}1 & \beta & \ldots & \beta^{d-1}\end{array}\right]$ is a left eigenvector for $\mathbf{C}$ with eigenvalue $\beta$, since

$$
\boldsymbol{\beta}^{T} \mathbf{C}=\left[\begin{array}{lll}
\beta & \beta^{2} \ldots & \beta^{d}
\end{array}\right]=\beta \boldsymbol{\beta}^{T}, \text { given that } \beta^{d}=-\sum_{k=0}^{d-1} \beta^{k} c_{k} .
$$

Definition 2.4: A real algebraic integer $\beta>1$ with Galois conjugates

$$
\beta_{2}, \ldots, \beta_{d}
$$

(a) is a Pisot number, if $\left|\beta_{i}\right|<1$ for every $2 \leq i \leq d$.

(b) is a Salem number, if $\left|\beta_{i}\right| \leq 1$ for every $2 \leq i \leq d$, and at least one pair of complex conjugate roots of $p(x)$ has modulus 1 .

The companion matrix of $p(x)$ induces an endomorphism in the $d$-torus $\mathbb{R}^{d} / \mathbb{Z}^{d}$. If $|\operatorname{det} \mathbf{C}|=\left|c_{0}\right|=1$ then $\mathbf{C} \in G L(d, \mathbb{Z})$ induces an automorphism in $\mathbb{R}^{d} / \mathbb{Z}^{d}$ (see [13, p. 46]). The minimal polynomial of a Pisot number induces an hyperbolic toral automorphism if and only if $\left|c_{0}\right|=1$. Such Pisot numbers are units in the ring $\mathbb{Q}[\beta]$. Any Salem number necessarily induces a (non-hyperbolic) toral automorphism, because its minimal polynomial is reciprocal and has even degree $d \geq 4$, therefore $c_{0}=1$ and $\operatorname{det} \mathbf{C}=1$.

\section{The companion map of the $\beta$-transformation}

Assume that $\beta>1$ is a real algebraic integer of degree $d \geq 2$, with minimal polynomial $p(x)$ and companion matrix C. $\mathbb{Q}(\beta)$ is a $\mathbb{Q}$-vector space of dimension $d$ and the coordinates of $\boldsymbol{\beta}:=\left(1, \beta, \ldots, \beta^{d-1}\right)$ form a basis (see [12, p. 429]). In this basis, the coordinate vector of $x \in \mathbb{Q}(\beta)$ is $\boldsymbol{x}:=\left(x_{0}, \ldots, x_{d-1}\right) \in \mathbb{Q}^{d}$, according to the isomorphism

$$
\begin{aligned}
\phi_{\boldsymbol{\beta}}: \mathbb{Q}^{d} & \longrightarrow \\
\boldsymbol{x} & \longmapsto x=\langle\boldsymbol{Q}(\beta) \\
& \longmapsto \boldsymbol{\beta}, \boldsymbol{x}\rangle
\end{aligned},
$$

where $\langle\boldsymbol{\beta}, \boldsymbol{x}\rangle=\sum_{k=0}^{d-1} \beta^{k} x_{k}$ is the inner product in $\mathbb{R}^{d}$, which can also be implemented in matrix form as $\boldsymbol{\beta}^{T} \boldsymbol{x}$ (or equivalently, $\boldsymbol{x}^{T} \boldsymbol{\beta}$ ). 
Let $\boldsymbol{e}_{1}:=(1,0, \ldots, 0)$ be the first vector of the standard basis of $\mathbb{R}^{d}$.

Proposition 3.1: Let $R:=\left\{\boldsymbol{x} \in \mathbb{Q}^{d} \mid 0 \leq\langle\boldsymbol{\beta}, \boldsymbol{x}\rangle \leq 1\right\}$ and $[\mathbf{C x}]:=\lfloor\langle\boldsymbol{\beta}, \mathbf{C} \boldsymbol{x}\rangle\rfloor \boldsymbol{e}_{1}=$ $\lfloor\beta x\rfloor \boldsymbol{e}_{1} \in \mathbb{Z}^{d}$. The companion map of the $\beta$-transformation is defined by

$$
\begin{aligned}
T_{\mathbf{C}}: R \subset \mathbb{Q}^{d} & \longrightarrow \quad R \subset \mathbb{Q}^{d} \\
\boldsymbol{x} & \longmapsto \mathbf{C x}-[\mathbf{C} \boldsymbol{x}]
\end{aligned}
$$

The companion map is conjugated by $\phi_{\boldsymbol{\beta}}$ to the $\beta$-transformation, that is

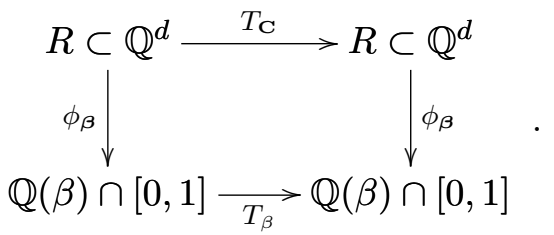

Proof. By definition, $\phi_{\boldsymbol{\beta}}$ is a bijection between $R \subset \mathbb{Q}^{d}$ and $\mathbb{Q}(\beta) \cap[0,1]$.

Let us check that the diagram is commutative. We have seen in (4) that $\boldsymbol{\beta}^{T}$ is a left eigenvector of $\mathbf{C}$, with eigenvalue $\beta$. Therefore $\langle\boldsymbol{\beta}, \mathbf{C} \boldsymbol{x}\rangle=\boldsymbol{\beta}^{T} \mathbf{C} \boldsymbol{x}=\beta \boldsymbol{\beta}^{T} \boldsymbol{x}=\beta\langle\boldsymbol{\beta}, \boldsymbol{x}\rangle$. Using this identity, we have

$$
\begin{aligned}
\phi_{\boldsymbol{\beta}} \circ T_{\mathbf{C}}(\boldsymbol{x}) & =\langle\boldsymbol{\beta}, \mathbf{C} \boldsymbol{x}-[\mathbf{C} \boldsymbol{x}]\rangle \\
& =\langle\boldsymbol{\beta}, \mathbf{C} \boldsymbol{x}\rangle-\langle\boldsymbol{\beta},[\mathbf{C} \boldsymbol{x}]\rangle \\
& =\beta\langle\boldsymbol{\beta}, \boldsymbol{x}\rangle-\lfloor\langle\boldsymbol{\beta}, \mathbf{C} \boldsymbol{x}\rangle\rfloor \\
& =\beta x-\lfloor\beta x\rfloor \\
& =T_{\beta} \circ \phi_{\boldsymbol{\beta}}(\boldsymbol{x}) .
\end{aligned}
$$

Remark: Proposition 3.1 is related to a reformulation in our setting of a familiar algebraic result. Namely, $\mathbb{Q}(\beta)$ is isomorphic, both as $\mathbb{Q}$-algebras and as $\mathbb{Q}[x]$-modules, to $\frac{\mathbb{Q}[x]}{<p(x)>}$. One defines the algebra homomorphism $\theta$ from $\mathbb{Q}[x]$ to $\mathbb{Q}(\beta)$ by sending $x$ to $\beta$ : this is clearly surjective, since $\mathbb{Q}(\beta)$ is spanned as a $\mathbb{Q}$-vector space by the powers of $\beta$. The kernel of $\theta$ is precisely $\langle p(x)\rangle$, given that $p(x)$ is the minimal polynomial of $\beta$. So, by the First Isomorphism Theorem for algebra homomorphisms, $\frac{\mathbb{Q}[x]}{\langle p(x)>} \cong \mathbb{Q}(\beta)$. This also shows that multiplication of an element of $\frac{\mathbb{Q}[x]}{\langle p(x)>}$ by $x$ corresponds to multiplication of the corresponding element of $\mathbb{Q}(\beta)$ by $\beta$. Finally, $\frac{\mathbb{Q}[x]}{\langle p(x)>}$ admits a $\mathbb{Q}$-basis $\left\{1, x, \ldots, x^{d-1}\right\}$, which defines an isomorphism between $\frac{\mathbb{Q}[x]}{<p(x)>}$ and $\mathbb{Q}^{d}$. The pair $\left(\mathbb{Q}^{d}, \mathbf{C}\right)$ becomes a $\mathbb{Q}[x]$-module if we define $x \cdot \mathbf{v}:=\mathbf{C} \mathbf{v}$ for $\mathbf{v} \in \mathbb{Q}^{d}$, hence multiplication by $\beta$ in $\mathbb{Q}(\beta)$ corresponds to the linear map $\mathbf{C}$ in $\mathbb{Q}^{d}$ (see [14, p. 478]).

It is known (see [13]) that $\mathbf{C}$ defines a decomposition

$$
\mathbb{R}^{d}=E^{u} \oplus E^{c} \oplus E^{s}
$$

where $E^{u}, E^{c}$ and $E^{s}$ are the unstable, central and stable subspaces associated to the eigenvalues of $\mathbf{C}$ with modulus greater than, equal or less than one. If $\beta$ is a Pisot number 


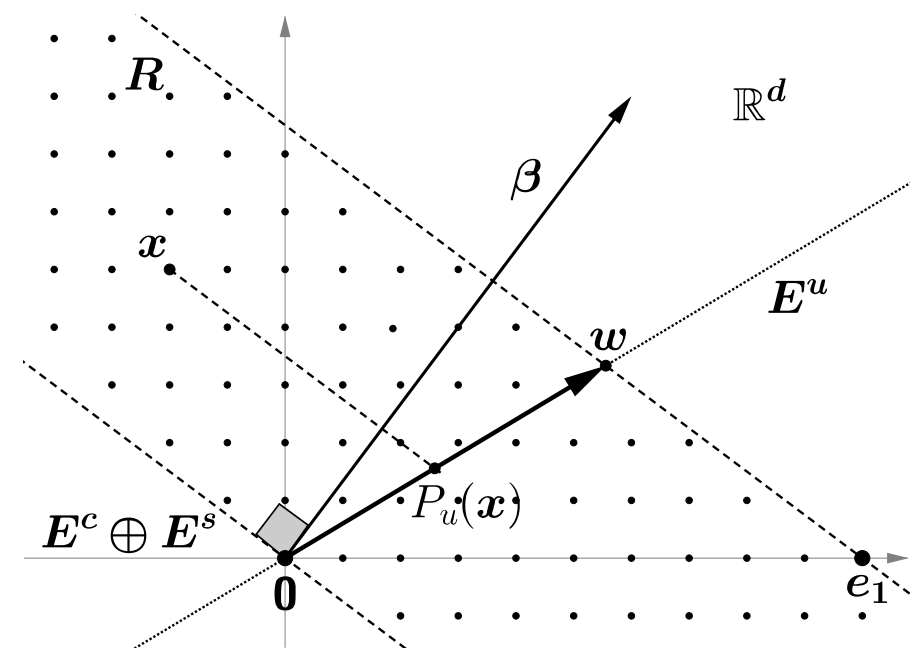

Figure 1. Domain of the Companion Map and Stable, Central and Unstable Subspaces

then $E^{c}=\varnothing$.

Proposition 3.2: If $\beta$ is a Pisot or a Salem number, then

(a) $E^{u}=\operatorname{ker}(\mathbf{C}-\beta \mathbf{I} \mathbf{d})$ is a subspace of dimension 1 .

(b) $E^{c} \oplus E^{s}=\operatorname{ker} \phi_{\boldsymbol{\beta}}$ is a subspace of dimension $(d-1)$.

Proof. If $\beta$ is a Pisot or a Salem number, then it is the only eigenvalue of $\mathbf{C}$ with modulus greater than 1 , therefore the $E^{u}$ is 1-dimensional and the complementary subspace $E^{c} \oplus$ $E^{s}$ must be $(d-1)$-dimensional.

Since $\boldsymbol{\beta}^{T} \mathbf{C}=\beta \boldsymbol{\beta}^{T} \Leftrightarrow \boldsymbol{\beta}^{T}(\mathbf{C}-\beta \mathbf{I} \mathbf{d})=\mathbf{0}_{1 \times d}$, then for any $\boldsymbol{x} \in \mathbb{R}^{d}$

$$
\boldsymbol{\beta}^{T}(\mathbf{C}-\beta \mathbf{I d}) \boldsymbol{x}=0 .
$$

If $\boldsymbol{x} \in E^{u}=\operatorname{ker}(\mathbf{C}-\beta \mathbf{I d})$ then (8) is a tautology. But if $\boldsymbol{x} \in E^{c} \oplus E^{s}$ then (8) implies that $(\mathbf{C}-\beta \mathbf{I d})\left(E^{c} \oplus E^{s}\right) \perp \boldsymbol{\beta}$. Since $(\mathbf{C}-\beta \mathbf{I} \mathbf{d})$ is an invertible linear map in $E^{c} \oplus E^{s}$, then $(\mathbf{C}-\beta \mathbf{I} \mathbf{d})\left(E^{c} \oplus E^{s}\right)=E^{c} \oplus E^{s}$, therefore $E^{c} \oplus E^{s} \perp \boldsymbol{\beta} \Leftrightarrow E^{c} \oplus E^{s}=\operatorname{ker} \phi_{\boldsymbol{\beta}}$.

$R \subset \mathbb{Q}^{d}$ is a region between $E^{c} \oplus E^{s}$ and $e_{1}+E^{c} \oplus E^{s}$, as shown in Figure 1.

Let $P_{u}: \mathbb{Q}^{d} \rightarrow E^{u} \subset \mathbb{R}^{d}$ be the projection onto the unstable space along hyperplanes parallel to $E^{c} \oplus E^{s}$. Define $\boldsymbol{w}:=P_{u}\left(\boldsymbol{e}_{1}\right)=E^{u} \cap\left(\boldsymbol{e}_{1}+E^{c} \oplus E^{s}\right)$, and

$$
\begin{aligned}
P_{u}(\boldsymbol{x}) & :=\phi_{\boldsymbol{\beta}}(\boldsymbol{x}) \boldsymbol{w} \\
& =\langle\boldsymbol{\beta}, \boldsymbol{x}\rangle \boldsymbol{w}
\end{aligned}
$$

If $\phi_{\boldsymbol{\beta}}(\boldsymbol{x})=x=\sum_{n=1}^{\infty} \frac{a_{n}}{\beta^{n}}$ then $P_{u}(\boldsymbol{x})=\sum_{n=1}^{\infty} \frac{a_{n}}{\beta^{n}} \boldsymbol{w}=\sum_{n=1}^{\infty} a_{n} \mathbf{C}^{-n} \boldsymbol{w}$. If $\beta$ is a Pisot unit, the projection of $\boldsymbol{w}$ into the $d$-dimensional torus is a fundamental homoclinic point for the toral automorphism induced by $\mathbf{C}$. This connects to the theory of arithmetic codings for hyperbolic automorphisms (see [10]) and symbolic representations of expansive group automorphisms (see [11]). 


\section{Periodic orbits, bounded orbits and Pisot numbers}

There exist $d$ embeddings $\sigma_{i}: \mathbb{Q}(\beta) \rightarrow \mathbb{C}$ such that $\sigma_{i}(\beta)=\beta_{i}$ (see [12]). Let us write the forward $T_{\beta}$ and $T_{\mathbf{C}}$ iterates as $x^{(n)}:=T_{\beta}^{n}(x)$ and $\boldsymbol{x}^{(n)}:=T_{\mathbf{C}}^{n}(\boldsymbol{x})$, and note that $x^{(n)}=\phi_{\boldsymbol{\beta}}\left(\boldsymbol{x}^{(n)}\right)$. If $\boldsymbol{\beta}_{i}:=\left(1, \beta_{i}, \ldots, \beta_{i}^{d-1}\right) \in \mathbb{C}^{d}$, then $\sigma_{i}\left(x^{(n)}\right)=\left\langle\boldsymbol{\beta}_{i}, \boldsymbol{x}^{(n)}\right\rangle$ and $\sigma_{1}$ is the inclusion map of $\mathbb{Q}(\beta)$ in $\mathbb{C}$. The crucial Lemma 2.3 of [6] can be reformulated as:

Theorem 4.1: Let $\beta:=\beta_{1}>1$ be an algebraic integer with Galois conjugates $\beta_{2}, \ldots, \beta_{d} \in \mathbb{C}$. If $x \in \mathbb{Q}(\beta) \cap[0,1], \boldsymbol{x} \in R \subset \mathbb{Q}^{d}$ and $\phi_{\boldsymbol{\beta}}(\boldsymbol{x}):=\langle\boldsymbol{\beta}, \boldsymbol{x}\rangle=x$, then the following statements are equivalent:

(a) $x \in \operatorname{Per}\left(T_{\beta}\right)$.

(b) $\boldsymbol{x} \in \operatorname{Per}\left(T_{\mathbf{C}}\right)$.

(c) $\exists B>0 \quad \forall n \in \mathbb{N}_{0}:\left\|\boldsymbol{x}^{(n)}\right\| \leq B$.

(d) $\exists K>0 \quad \forall 1 \leq i \leq d \forall n \in \mathbb{N}_{0}:\left|\left\langle\boldsymbol{\beta}_{i}, \boldsymbol{x}^{(n)}\right\rangle\right| \leq K$.

Proof. $\phi_{\boldsymbol{\beta}}$ conjugates $\left(R, T_{\mathbf{C}}\right)$ and $\left(\mathbb{Q}(\beta) \cap[0,1], T_{\beta}\right)$ therefore $(a) \Leftrightarrow(b)$.

If $\boldsymbol{x} \in \operatorname{Per}\left(T_{\mathbf{C}}\right)$ then $\left\{\boldsymbol{x}^{(n)}\right\}$ is a finite (bounded) set, thus $(b) \Rightarrow(c)$. To prove the converse, suppose $(c)$ holds. Since $T_{\mathbf{C}}$ is the composition of the linear map with integer coefficients $\mathbf{C}$ with a translation in $\mathbb{Z}^{d}$, then $\left\{\boldsymbol{x}^{(n)}\right\}$ is a subset of the lattice $\frac{1}{q} \mathbb{Z}^{d}$, where $q$ is the m.c.d. of $\boldsymbol{x}$ 's coordinates. A bounded subset of a lattice is finite, therefore $(c) \Rightarrow(b)$. If (c) holds, then (d) is true, because

$$
\left|\left\langle\boldsymbol{\beta}_{i}, \boldsymbol{x}^{(n)}\right\rangle\right| \leq\left\|\boldsymbol{\beta}_{i}\right\| \cdot\left\|\boldsymbol{x}^{(n)}\right\| \leq \max _{1 \leq i \leq d}\left\{\left\|\boldsymbol{\beta}_{i}\right\|\right\} \cdot B=K
$$

Finally, following an idea from [6, p. 272], we have

$$
\left[\begin{array}{c}
\left\langle\boldsymbol{\beta}_{1}, \boldsymbol{x}^{(n)}\right\rangle \\
\left\langle\boldsymbol{\beta}_{2}, \boldsymbol{x}^{(n)}\right\rangle \\
\vdots \\
\left\langle\boldsymbol{\beta}_{d}, \boldsymbol{x}^{(n)}\right\rangle
\end{array}\right]=\underbrace{\left[\begin{array}{cccc}
1 & \beta_{1} & \cdots & \beta_{1}^{d-1} \\
1 & \beta_{2} & \cdots & \beta_{2}^{d-1} \\
\vdots & \vdots & \cdots & \vdots \\
1 & \beta_{d} & \cdots & \beta_{d}^{d-1}
\end{array}\right]}_{V} \boldsymbol{x}^{(n)} .
$$

If $(d)$ is true, then left hand side of (10) is a bounded vector for every $n \in \mathbb{N}_{0}$. Since $V$ is a non-singular Vandermonde matrix $\left(\beta_{i} \neq \beta_{j}\right.$ for $\left.i \neq j\right)$, then $\boldsymbol{x}^{(n)}$ is a bounded vector (for every $n \in \mathbb{N}_{0}$ ) and we obtain (c).

The following theorem is a classic result of [6, p. 274] and [5], which we reproduce for the sake of completeness.

Theorem 4.2: Let $\beta$ be a Pisot number. Then $\operatorname{Per}\left(T_{\beta}\right)=\mathbb{Q}(\beta) \cap[0,1]$.

Proof. This is a reformulation in our setting of the proof in [6, p. 274].

Let $x \in \mathbb{Q}(\beta) \cap[0,1]$ and write its $T_{\beta}$-iterates as

$$
x^{(n)}=\beta^{n}\left(x-\sum_{k=1}^{n} \frac{a_{k}}{\beta^{k}}\right),
$$

where $\left(a_{1}, a_{2}, \ldots\right)$ is the $\beta$-expansion of $x$ and $0 \leq a_{k} \leq\lfloor\beta\rfloor$. 
Applying $\sigma_{i}$ to both members of this equation, we obtain

$$
\left\langle\boldsymbol{\beta}_{i}, \boldsymbol{x}^{(n)}\right\rangle=\beta_{i}^{n}\left(\left\langle\boldsymbol{\beta}_{i}, \boldsymbol{x}\right\rangle-\sum_{k=1}^{n} \frac{a_{k}}{\beta_{i}^{k}}\right) .
$$

By definition $\beta_{1}:=\beta$, and it follows that $\left|\left\langle\boldsymbol{\beta}_{1}, \boldsymbol{x}^{(n)}\right\rangle\right|=\left|x^{(n)}\right| \leq 1$. If $2 \leq i \leq d$ then $\left|\beta_{i}\right|<1$, because $\beta$ is a Pisot number, therefore

$$
\begin{aligned}
\left|\left\langle\boldsymbol{\beta}_{i}, \boldsymbol{x}^{(n)}\right\rangle\right| & \leq\left|\beta_{i}\right|^{n} \cdot\left|\left\langle\boldsymbol{\beta}_{i}, \boldsymbol{x}\right\rangle\right|+\lfloor\beta\rfloor \cdot \sum_{k=1}^{n}\left|\beta_{i}\right|^{n-k} \\
& \leq \max _{i}\left|\left\langle\boldsymbol{\beta}_{i}, \boldsymbol{x}\right\rangle\right|+\lfloor\beta\rfloor \cdot\left(1-\left|\beta_{i}\right|\right)^{-1} \leq K .
\end{aligned}
$$

This proves (d) of Theorem 4.1, which is equivalent to $x \in \operatorname{Per}\left(T_{\beta}\right)$.

The dynamical systems $\left(R, T_{\mathbf{C}}\right)$ and $\left(\mathbb{Q}^{d} / \mathbb{Z}^{d}, \bar{C}\right)$, where $\bar{C}$ is the toral endo/automorphism induced by $\mathbf{C}$, are semi-conjugated by

$$
\begin{aligned}
\pi: \mathbb{Q}^{d} & \longrightarrow \mathbb{Q}^{d} / \mathbb{Z}^{d} \\
\boldsymbol{x} & \longmapsto \boldsymbol{x}+\mathbb{Z}^{d}
\end{aligned}
$$

since $\pi$ is a continuous surjection and the following diagram commutes

$$
\begin{gathered}
R \subset \mathbb{Q}^{d} \stackrel{T_{\mathbf{C}}}{\longrightarrow} R \subset \mathbb{Q}^{d} \\
\mathbb{Q}^{d} / \mathbb{Z}^{d} \longrightarrow \underset{v^{\prime}}{\longrightarrow} \mathbb{Q}^{d} / \mathbb{Z}^{d} \\
\pi \circ T_{\mathbf{C}}(\boldsymbol{x})=\pi(\mathbf{C} \boldsymbol{x}-[\mathbf{C} \boldsymbol{x}])=\mathbf{C} \boldsymbol{x}+\mathbb{Z}^{d}=\bar{C} \circ \pi(\boldsymbol{x}) .
\end{gathered}
$$

The period of $\boldsymbol{x}$ for $T_{\mathbf{C}}$ is a multiple of the period of $\pi(\boldsymbol{x})$ for $\bar{C}$. This is the reformulation in our setting of Proposition 3.3. from [6, p. 275].

Theorem 3.4. of [6, p. 275] proves that if $\beta$ is a Pisot number with minimal polynomial $p(x)=x^{2}-n x-1$, for some $n \in \mathbb{N}$, then every $x \in \mathbb{Q}(\beta) \cap[0,1]$ has strictly periodic $\beta$-expansion. It is also claimed that the period of $x$ for $T_{\beta}$ is the same as the period of $\pi(\boldsymbol{x})$ for $\bar{C}$. We note that this is the only case where the companion matrix is symmetric

$$
\mathbf{C}=\left[\begin{array}{ll}
0 & 1 \\
1 & n
\end{array}\right]
$$

hence $\mathbb{R}^{2}=E^{u} \oplus E^{s}$ is an orthogonal decomposition. It would be interesting to pursue the study of this particular case, and find out what distinguishes it from the general Pisot cases. 


\section{Conclusion}

When $\beta$ is a Pisot or a Salem number of degree $d>1$, the $\beta$-transformation is conjugate to a transformation on a subset of $\mathbb{Q}^{d}$. We call it the companion map for the $\beta$-transformation, because its definition is related to the companion matrix of the minimal polynomial of $\beta$. This setting emphasizes the Dynamical Systems' point of view, rather than the original number theoretical framework.

The companion map and the associated notation provide a good framework to reformulate the classical result from [6] concerning the set of eventually periodic points of the $\beta$-transformation when $\beta$ is a Pisot number. Furthermore, it gives a geometric representation of the orbits of the $\beta$-transformation in a $d$-dimensional space, which could yield insight useful for further study of this area.

\section{Acknowledgement(s)}

I would like to thank Anthony Manning for his suggestions concerning this work over a long period of time. I'm also thankful to Pedro Miguel Duarte for his encouragement and feedback concerning the preliminary drafts. I would like to thank Kenneth A. Brown for his suggestions concerning the remark to Proposition 3.1.

Last but not the least, I would like to thank my good friend Daniel Thompson, whose knowledge and support was invaluable towards the completion of this work.

\section{References}

[1] Renyi A. Representations of real numbers and their ergodic properties. Acta Math Hungar. 1957;8:477-493.

[2] Parry W. On the $\beta$-expansions of real numbers. Acta Math Hungar. 1960;11:401-416.

[3] Maia B. An equivalent system for studying periodic points of the beta transformation for a Pisot or a Salem number [dissertation]. Warwick; 2008.

[4] Climenhaga V, Thompson D. Intrinsic ergodicity beyond specification: $\beta$-shifts, $s$-gap shifts, and their factors. Isr J Math. 2012;192, Part B:785-817.

[5] Bertrand A. Développements en base de Pisot et répartition modulo 1. C R Acad Sci Paris. 1977;Sér. A-B, 285 (6):419-421.

[6] Schmidt K. On periodic expansions of Pisot numbers and Salem numbers. Bull London Math Soc. $1980 ; 12: 269-278$.

[7] Boyd D. Salem numbers of degree four have periodic expansions. In: de Gruyter BNY, editor. Théorie des nombres (Conference Proceedings, Banff); 1988. p. 57-64.

[8] Boyd D. On the beta expansion for Salem numbers of degree 6. Math Comp. 1996;65:861875.

[9] Thurston W. Entropy in dimension one. 2014.

[10] Sidorov N, Vershik A. Bijective arithmetic codings of the 2-torus, and binary quadratic forms. J Dynam Cont Sys. 1998;4:365-400.

[11] Lindenstrauss E, Schmidt K. Symbolic representations of nonexpansive group automorphisms. Isr J Math. 2005;149:227-266.

[12] Birkhoff G, MacLane S. A survey of modern Algebra. Macmillan Publishing Co.; 1977.

[13] Katok A, Hasselblatt B. Introduction to the modern theory of dynamical systems. Cambridge University Press; 1995.

[14] Artin M. Algebra. Prentice Hall; 1991. 